ARTICLE

Received 4 May 2011 | Accepted 8 Oct 2012 | Published 13 Nov 2012 DOl: 10.1038/ncomms2192

\title{
Genome sequences of wild and domestic bactrian camels
}

The Bactrian Camels Genome Sequencing and Analysis Consortium*

Bactrian camels serve as an important means of transportation in the cold desert regions of China and Mongolia. Here we present a $2.01 \mathrm{~Gb}$ draft genome sequence from both a wild and a domestic bactrian camel. We estimate the camel genome to be $2.38 \mathrm{~Gb}$, containing 20,821 protein-coding genes. Our phylogenomics analysis reveals that camels shared common ancestors with other even-toed ungulates about 55-60 million years ago. Rapidly evolving genes in the camel lineage are significantly enriched in metabolic pathways, and these changes may underlie the insulin resistance typically observed in these animals. We estimate the genome-wide heterozygosity rates in both wild and domestic camels to be $1.0 \times 10^{-3}$. However, genomic regions with significantly lower heterozygosity are found in the domestic camel, and olfactory receptors are enriched in these regions. Our comparative genomics analyses may also shed light on the genetic basis of the camel's remarkable salt tolerance and unusual immune system.

Correspondence and requests for materials should be addressed to J. (email: yeluotuo@imau.edu.cn) or to Y.L. (email: yxli@sibs.ac.cn) or to H.M. (email: menghe@sjtu.edu.cn).

${ }^{*}$ A full list of The Bactrian Camels Genome Sequencing and Analysis Consortium and their affiliations appears at the end of the paper. 
W ild bactrian camels (Camelus bactrianus ferus) are the lone survivors of the old world camels ${ }^{1}$. At present, their total number is only $730-880$, less than that of the giant pandas ${ }^{2}$. They live in northwestern China and southwestern Mongolia, especially the Outer Altai Gobi Desert. Considered critically endangered by the International Union for Conservation of Nature, wild bactrian camels are protected under both the Convention on International Trade in Endangered Species of Wild Fauna and Flora and domestic legislations in China and Mongolia. The archaeozoological record shows that fully domesticated bactrian camels were present in the third millennium BC and subsequently spread into much of Central Asia $^{3}$. However, our knowledge about the origins and migration history of domestic camels remains inconclusive.

To adapt to the harsh conditions-cold, hot, arid, and poor grazing-of deserts or semi-deserts, camels have acquired many special abilities and attributes. They can store energy in their humps and abdomen in the form of fat, enabling them to survive long periods without any food or water ${ }^{4}$. The camel's body temperature may vary from 34 to $41^{\circ} \mathrm{C}$ throughout the day ${ }^{5}$. Blood glucose levels in camels are twice those of other ruminants ${ }^{6}$. Camels tolerate a high dietary intake of salt, consuming eight times more than cattles and sheep ${ }^{7}$, yet they do not develop diabetes or hypertension. The Camelidae family are the only mammals that can produce heavy-chain antibodies (HCAbs), a special form of immunoglobulin that lacks the light chain, in contrast to conventional antibodies (Abs) ${ }^{8}$. HCAbs are smaller and more stable, offering particular advantages in various medical and biotechnological applications.

In this study, we sequenced the genomes of both wild and domestic bactrian camel, to better understand the history of their evolution and domestication, and to provide a resource for research into the genetic mechanisms that enable camels to survive extreme environments.

\section{Results}

Genome sequence. We sequenced the genomes of an 8-year-old wild male bactrian camel named 'Naran' from the wild bactrian camel nature reserve of Altai province, Mongolia ('wild camel' hereafter, Supplementary Fig. S1) and a 6-year-old male Alashan bactrian camel from Inner Mongolia, China ('domestic camel' hereafter, Supplementary Fig. S2). For the wild camel genome, four paired-end/mate-pair sequencing libraries were constructed with insert sizes of $500 \mathrm{bp}, 3 \mathrm{~kb}, 10 \mathrm{~kb}$ and $20 \mathrm{~kb}$. For the domestic camel genome, only libraries with shorter insert size of $500 \mathrm{bp}$ were constructed (Supplementary Table S2). We assembled the short reads obtained from the wild camel genome sequencing using SOAPdenovo 9 . The reads with the insert size of $500 \mathrm{bp}$ were first assembled into contigs. Then the contigs were joined into scaffolds with reads from the shortest to the longest insert size. In total, we obtained 120,352 scaffolds, including 13,544 scaffolds longer than $1 \mathrm{~kb}$ and 3,453 longer than $10 \mathrm{~kb}$. The N50 length of the scaffolds longer than $1 \mathrm{~kb}$ is $2.00 \mathrm{Mb}$ (Table 1 ). We remapped the usable reads to the scaffolds and obtained an average effective depth of $76 \times$ and $24 \times$ for the wild and the domestic camel genomes, respectively (Supplementary Table S3). Using the frequency distribution of 17-mer in the reads (Supplementary Fig. S3), we estimated the camel genome size to be $2.38 \mathrm{~Gb}$. This is close to the camel genome size $(2.02-2.40 \mathrm{~Gb})$ calculated based on haploid DNA contents ( $C$ values) (Supplementary Table S4).

The genome sequences show that $34 \%$ of the bactrian camel genome are repetitive DNAs (Supplementary Table S5). This percentage is lower than that in human $(>50 \%)^{10}$, horse $(46 \%)^{11}$ or cattle $(48 \%)^{12}$, but close to that in mouse $(35 \%)^{13}$ or dog $(34 \%)^{14}$. Most of the repetitive DNAs are transposon-derived repeats. Long interspersed elements cover $19 \%$ of the whole genome, comparable to human (21\%), mouse (19\%), horse (20\%) and cattle $(23 \%)$. In contrast, the percentage of short interspersed elements is lower in bactrian camel (4\%) than in human (13\%), mouse $(8 \%)$, horse $(7 \%)$ and cattle $(18 \%)$. This is likely one of the reasons that the bactrian camel has a smaller genome size than other mammals, for example, human $(2.9 \mathrm{~Gb})^{10}$, mouse $(2.5 \mathrm{~Gb})^{13}$, horse $(2.7 \mathrm{~Gb})^{11}$ and cattle $(2.9 \mathrm{~Gb})^{12}$. Especially, although copies of ALU repeats appear frequently in primate genomes $^{15}$, none exist in the bactrian camel genome. In addition, 244,141 simple repeats (microsatellite) loci were identified in the camel genome (Supplementary Table S5), which should be useful in quantitative trait locus mapping or marker assistant selection in camels.

Gene content and annotation. We annotated the camel genome using two $a b$ initio gene finders: Augustus ${ }^{16}$ and GenScan ${ }^{17}$. We also utilized the homology-based method, comparing it with several other mammalian genomes, including human, chimpanzee, mouse, rat, dog, horse and cattle, as well as the published expressed sequence tag data ${ }^{18}$ of dromedary camels. Combining these two methods, we predicted 20,821 bactrian camel genes, averaging eight exons and 1,322 bps coding region (CDS) per gene. Notably, the GC content of the CDS region is $52 \%$, significantly higher than that of the whole genome (41\%). Similar differences were observed in other mammals such as pig (50\% versus $40 \%)^{19}$.

Among the camel genes, 12,050 were annotated to at least one term in Gene Ontology $(\mathrm{GO})^{20}$ (Supplementary Fig. S4), and 4,750 genes were annotated to 288 KEGG pathways ${ }^{21}$. According to the InterProScan ${ }^{22}$ annotation (Supplementary Fig. S5), the most common protein domains found in the camel genome are immunoglobulin-like domains, consistent with a previous report ${ }^{18}$. The largest protein family identified from the camel genome is the rhodopsin-like G protein-coupled receptor family, which, with 1,011 members, is well-known for controlling the signalling pathways of many biological and physiological processes such as feeding, reproduction and behaviour.

Genome evolution. HomoloGene $e^{23}$ was used to examine the conservation of gene repertoires among bactrian camels and other vertebrate species. A total of 16,065 camel genes were grouped into 12,536 orthlogous families, of which 12,521 genes are conserved in vertebrates and 2,912 in mammals (Fig. 1a). A total of 4,756 unique genes were found in bactrian camels, among which 3,774 genes do not have GO annotations. Using the identified orthologs as anchor points, we constructed the syntenic maps between the camel and other mammalian genomes (Supplementary Table S6). In total, we identified more than 1,100 syntenic blocks in the camel genome, which cover 12,965 orthologous camel genes (Fig. 1b).

We constructed a phylogenetic tree with the supertree method $^{24}$, using 2,345 single-copy orthologs among the animals (Fig. 1c). As shown in this tree, cattle and pig are the closest relatives of bacterian camels. All of them belong to Artiodactyla (even-toed ungulates) in taxonomy, which form a sister group with the clades of Perissodactyla (for example, horse) and Carnivora (for example, dog). This phylogeny was also consistent with previous evolutionary studies based on a smaller number of camel genes ${ }^{25}$. Among the single-copy orthologs, we further selected 332 orthologs with constant evolutionary rates to determine the time of speciation events. We estimated that cattle and bactrian camel lineages diverged about 55-60 million years ago (Mya) (Fig. 1c and Supplementary Fig. S6), in the later 
Table 1 | Statistics of wild camel genome assembly.

Large scaffolds ( $\geq 1,000 \mathrm{bp})$

Contigs ( $\geq 200$ bp)

No. of large scaffolds

Largest length

Bases in large scaffolds

N50 scaffold length

N50 scaffold index

N90 scaffold length

N90 scaffold index

GC content (\%)

$\mathrm{N}$ rate $(\%)$

13,544
$15,735,958$
$2,010,007,732$
$2,005,940$
274
341,009
1,169
41.28
1.18

73,065

853,441

$1,985,490,767$

85,292

6,136

15,299

26,984

41.28

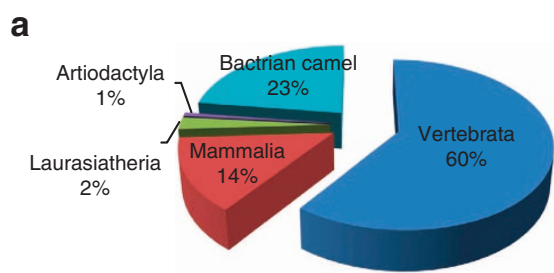

b

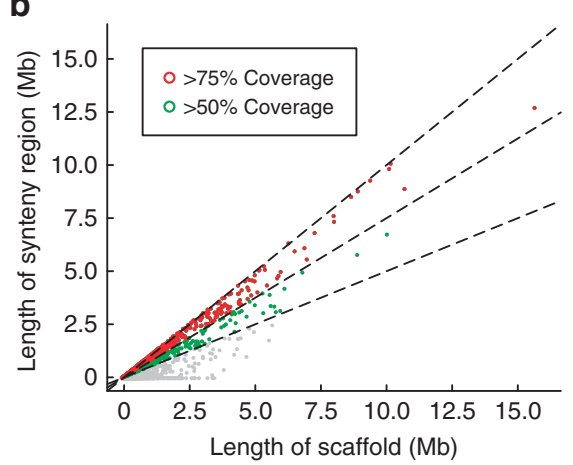

c

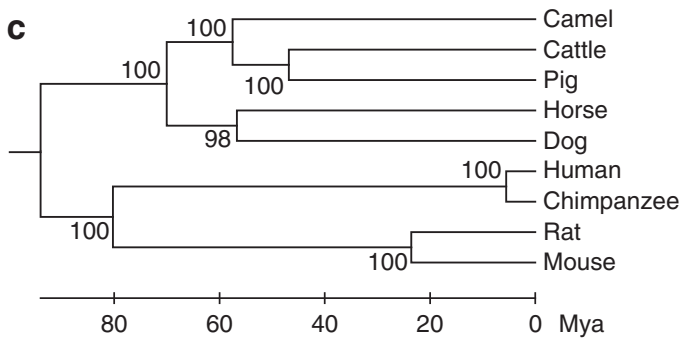

d

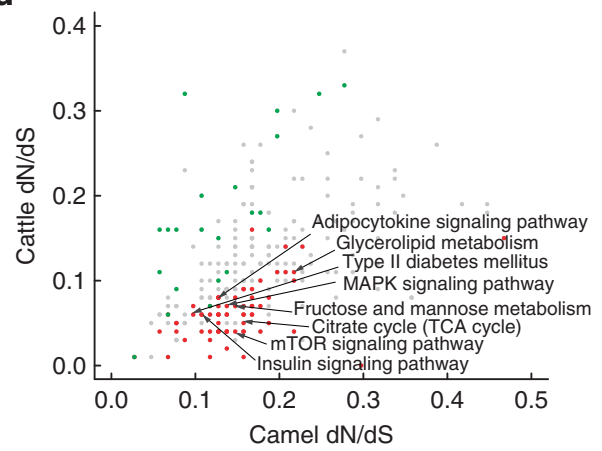

Figure 1 | Genomic comparison between bactrian camel and other animals. (a) Proportion of shared orthologs between bactrian camel and animals in Vertebrata (chicken and zebrafish; NCBI genome accession codes GCF_000002315.3 and GCF_000002035.4), Mammalia (human, chimpanzee, mouse and rat; NCBI genome accession codes GCF_000001405.21, GCF_000001515.5, GCF_000001635.20 and GCF_000001895.4), Laurasiatheria (dog and horse; NCBI genome accession codes GCF_000002285.3 and GCF_000002305.2) and Artiodactyla (cattle and pig; NCBI genome accession codes GCF_000003055.4 and GCF_000003025.5). (b) Length of syntenic regions on each scaffold (Mb, million base pairs). Coverage is calculated as the length of syntenic region divided by the length of scaffold. Scaffolds with coverage $>75 \%,>50 \%$ and $<50 \%$ are represented by red, green and grey dots, respectively. (c) Supertree inference for nine mammals. The topology was evaluated by input tree bootstrap percentages. Distances are shown in millions of years. (d) Points represent pairs of medians of $\mathrm{dN} / \mathrm{dS}$ ratios in camels and in cattle by KEGG pathways. Pathways in which rapidly evolving genes are significantly enriched $(F D R<0.05)$ in camels and cattle are coloured in red and green, respectively. MAPK, mitogen-activated protein kinase; mTOR, mammalian target of rapamycin; TCA, tricarboxylic acid cycle.

Palaeocene period (55.8-65.5 Mya). This is slightly earlier than the first fossil evidence of the Camelidae family in North America (50 Mya) ${ }^{26}$.

Rapidly evolving genes. Rapid divergence of protein-coding genes, as measured by an increased ratio of nonsynonymous-tosynonymous substitutions $(\mathrm{dN} / \mathrm{dS})$, may have important roles in species differentiation and adaption ${ }^{27,28}$. We estimated the $\mathrm{dN} / \mathrm{dS}$ ratios by the PAML package ${ }^{29}$ for the camel and its closest cattle orthologs, taking the human ortholog as an outgroup. We used the likelihood ratio test (LRT) to identify 2,730 significantly faster evolving genes in camels than in cattle (false discovery rate $($ FDR $)<0.05)$ and mapped them to the KEGG pathways (Supplementary Table S7). It was shown that those rapidly evolving genes are significantly enriched in carbohydrate metabolism, lipid metabolism and signalling pathways regulating the metabolic processes, such as insulin $\left(\right.$ FDR $\left.=9.1 \times 10^{-4}\right)$ and adipocytokine signalling pathways $(F D R=0.03$, Fig. $1 \mathrm{~d})$. The accelerated evolution of these pathways involved in metabolism may help camels to optimize their energy storage and production in the desert.

Characterization of heterozygosity. We identified 1,986,420 heterozygous single nucleotide polymorphisms (SNPs) in the wild camel genome and 2,129,442 heterozygous SNPs in the domestic camel genome (Supplementary Table S8). In both cases, the heterozygosity rates are estimated to be about $1.0 \times 10^{-3}$ across the whole genomes (Fig. 2a). The number of small indels identified in the two genomes is also comparable (Supplementary Table S9). 
a

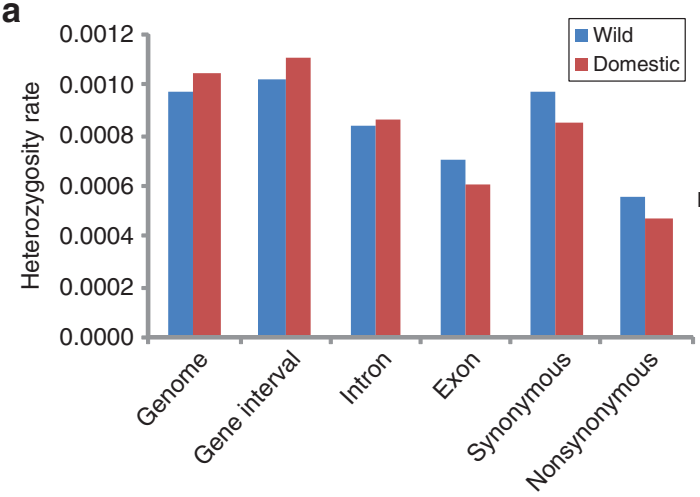

c

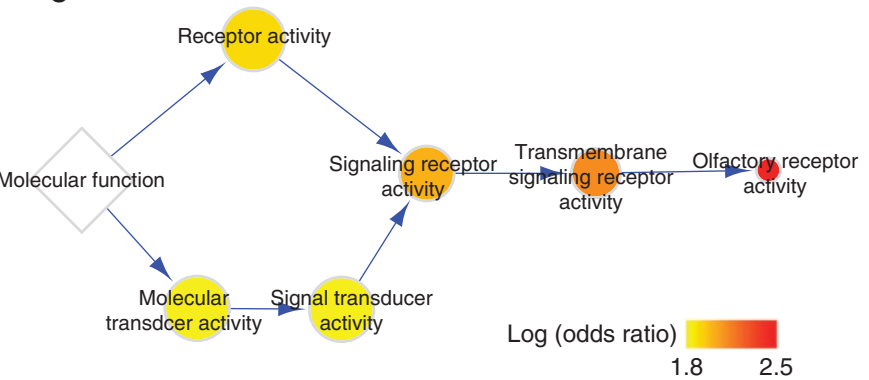

b

Wild depth Wild SNP

Scaffold 351

Domestic SNP

Domestic depth

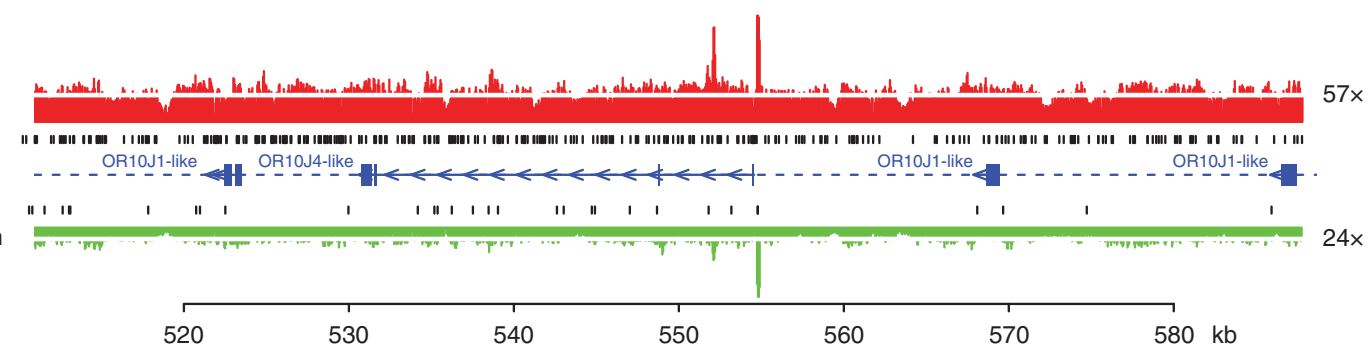

Figure 2 | Comparison of genetic diversity between wild and domestic bactrian camels. (a) Heterozygosity rate in coding and non-coding regions.

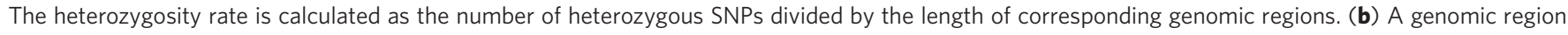
where the heterozygosity of the domestic camel is significantly lower than that of the wild one. The region also contains a cluster of olfactory receptors (OR10J1, olfactory receptor 10J1). Genes and gene intervals are represented by solid and dash lines, respectively. Exons are shown in blue blocks and

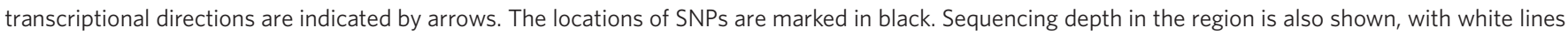
indicating the average sequencing depth. (c) Enrichment of molecular function for genes with low heterozygosity in the domestic camel. The hierarchy of the Gene Ontology is displayed. The size of the circle is proportional to the number of genes in the genome, and the colour indicates the odds ratio of the enrichment.

We then classified the SNPs according to the gene annotations and calculated the heterozygosity rates for coding and noncoding regions (Fig. 2a and Supplementary Table S10). Comparing with the wild camel, we found that an overall lower heterozygosity rate exists in the exon regions but not in other parts of the domestic camel genome, suggesting an artificial selection for certain genes in the domestic species ${ }^{30}$. As strong artificial selection would reduce genetic diversity around a locus (selective sweep) $)^{31}$, it was worth inspecting the genes and their functions in such a locus. We therefore used 10-kb windows to scan the genome to identify regions where the heterozygosity rate of the domestic camel is significantly lower than the wild one $\left(P<0.05\right.$ after Bonferroni correction, $\chi^{2}$-test $)$ (Fig. $\left.2 \mathrm{~b}\right)$. There are 2,816 such regions identified, which incorporate 196 complete genes (Supplementary Table S11). GO analysis of these genes showed that they are significantly enriched in membrane receptors and signalling transduction, and more specifically, olfactory receptor activity $\left(\mathrm{FDR}=3.8 \times 10^{-15}\right)$ (Fig. $2 \mathrm{c}$ and Supplementary Table S12). All of the olfactory receptors identified here (37 genes in total) are distributed in 17 different large scaffolds with median length over $800 \mathrm{~kb}$ (Supplementary Table S11), implying that the loci for the olfactory receptors are independent in genealogy. Therefore, it is reasonable to compare the heterozygosity rates of these loci between the wild and domestic camels, even though we only obtained data from one individual of each type. These results suggested that olfaction may be an important object of artificial selection during the domestication of bactrian camels.

Blood glucose levels. In general, blood glucose levels in domestic ruminants $\left(2.5-3.5 \mathrm{mmoll}^{-1}\right)$ are lower than in monogastric animals $\left(3.5-5.0 \mathrm{mmoll}^{-1}\right)^{32,33}$. Although camels belong to the suborder Tylopoda within Artiodactyla, they are also ruminating herbivores with an extensive forestomach. The levels of blood glucose in camels $\left(6-8 \mathrm{mmoll}^{-1}\right)$, however, are much higher than in most monogastrics ${ }^{32,33}$. Previous physiological experiments demonstrated that the high level of blood glucose in camels may be caused by their strong capacity for insulin resistance ${ }^{33}$. Consistent with this argument, our analysis shows that a large number of rapidly evolving genes in camels are involved in Type II diabetes mellitus (KEGG pathway accession code 04930 ) and the insulin signalling pathway (KEGG pathway accession code 04910) (Fig. 1d). The binding of insulin (INS) to insulin receptors could lead to tyrosine phosphorylation of insulin receptor substrates (IRSs), which will in turn activate PI3K and AKT to trigger downstream actions to promote glucose uptake and storage $e^{34,35}$ (Fig. 3a). Of particular note was that PI3K and $A K T$, two critical genes in the process, have undergone rapid divergence in camels, which may change their responsiveness to insulin. Several other rapidly evolving genes, such as $J N K, I K K$, $m T O R$ and $E R K$, could also result in insulin resistance via serine phosphorylation of IRS proteins to negatively regulate IRS activities $^{34,35}$ (Fig. 3a).

Cytochrome P450 families. Genes in cytochrome P450 (CYP) families are involved in the metabolism of arachidonic acids (KEGG pathway accession code 00590). For the CYP genes that were identified by InterProScan in the camel genome, we further assigned them to subfamilies by searching against the KEGG protein database and NCBI NR database. We found that the distribution of CYP genes in several subfamilies is quite different between the camel and other mammals (Supplementary 
a

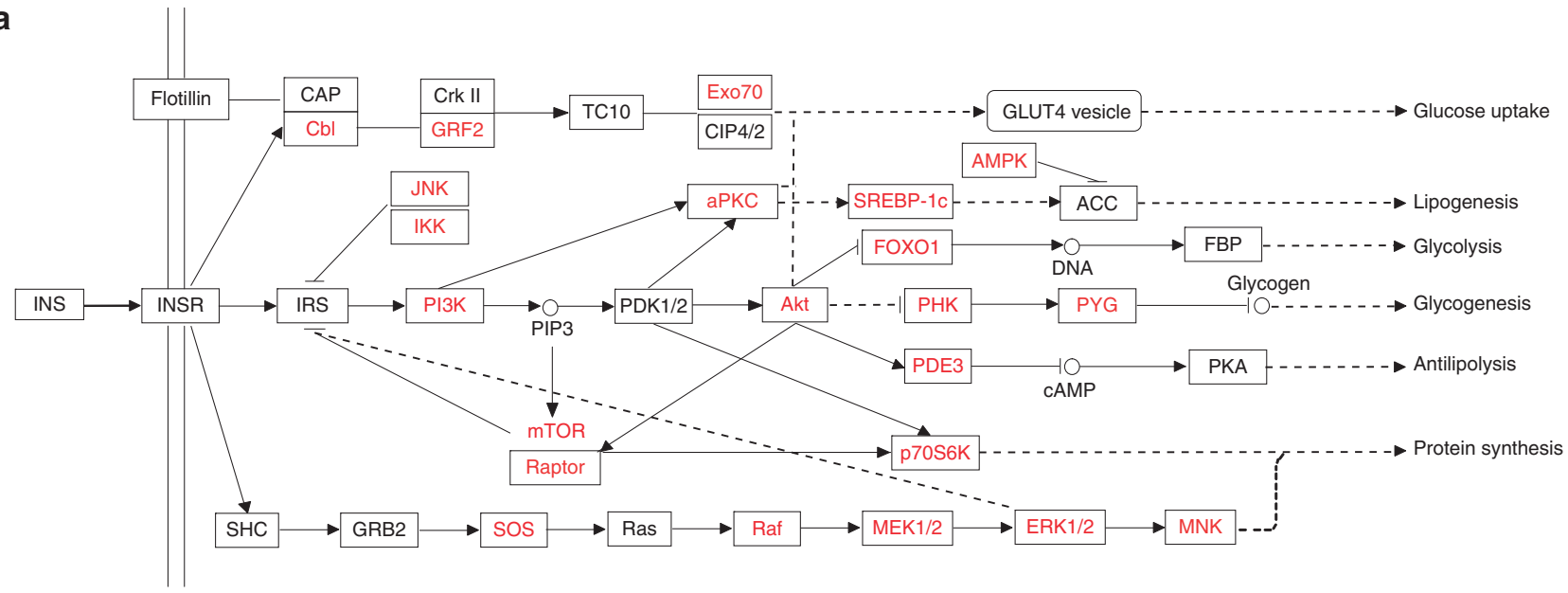

b

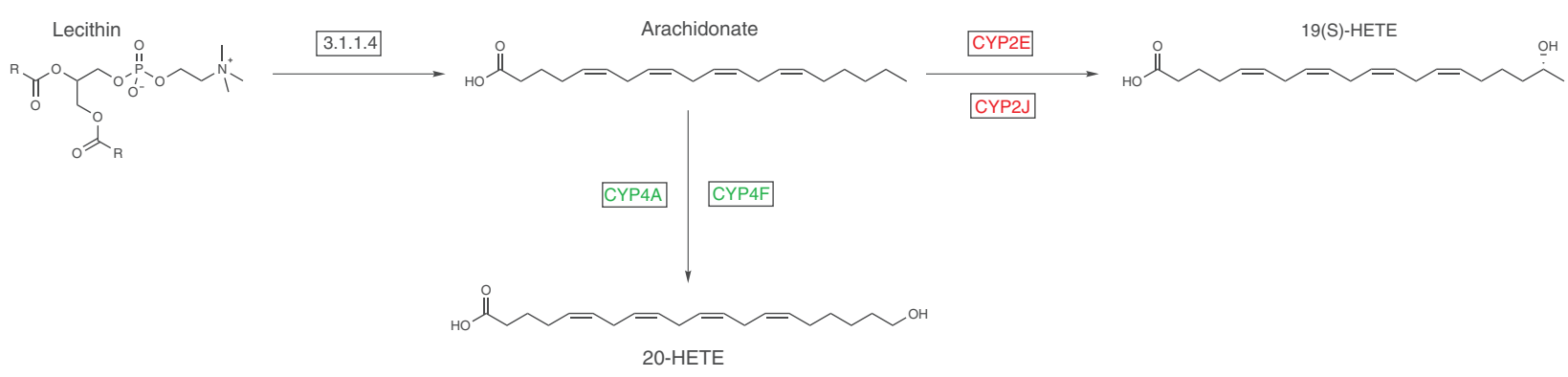

C

VH VH

DH

$\mathrm{JH}$

$\mathrm{C} \mu$

$\mathbf{C} \delta$

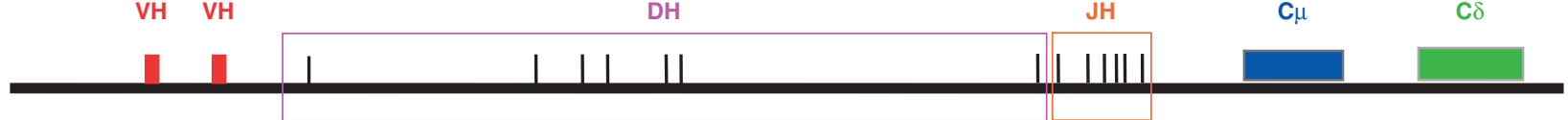

Figure 3 | Biological findings from the genome analysis. (a) The type II diabetes mellitus and insulin signalling pathway. The rapidly evolving genes in camels (shown in red) are identified in the pathway. Abbreviations, annotations and connexions are presented in accordance with KEGG standards: solid lines represent direct relationships among proteins (boxes) and metabolites (circular nodes), dashed lines represent indirect relationships, lines with arrowheads denote activation, and lines with the crossing mean inhibition. (b) The pathway of arachidonate synthesis and conversion. Arachidonate is synthesized from lecithin by PLA2G (EC: 3.1.1.4), and converted into 19(S)-hydroxyeicosatetraenoic acid (19(S)-HETE) by CYP2E and CYP2J (expansion in camel), or transformed into 20-hydroxyeicosatetraenoic acid (20-HETE) by CYP4A and CYP4F (contraction in camel). (c) The schematic diagram of IgH loci in the camel genome inferred from a complete V-D-J-C gene cluster in scaffold 355.1.

Table S13). In bactrian camels, there are 11 copies of CYP2J and 2 copies of CYP2E, more than in cattle (four and one, respectively), horses (one and one) and humans (one and one). In contrast, there are only one copy of CYP4A and two copies of CYP4F in camels, fewer than in cattle (three and seven, respectively), horses (three and seven) and humans (two and six). CYP2E and CYP2J can help to transform arachidonic acid into 19(S)-HETE, whereas CYP4F and CYP4A help to transform it into 20-HETE (Fig. 3b). 19(S)-HETE has been demonstrated to be a potent vasodilator of renal preglomerular vessels that stimulate water reabsorption ${ }^{36}$. So more copies of CYP2E and CYP2J and fewer copies of CYP4A and CYP4F may help camels produce more 19(S)-HETE, potentially useful for survival in the desert. In addition, the activity of CYP2J2 is regulated by high-salt diet and its suppression can lead to high blood pressure ${ }^{37}$. Camels are known to be able to take in a large amount of salt apparently without developing hypertension, perhaps because they have more copies of CYP2J genes.

Heavy-chain antibodies. A HCAb is an immunoglobulin that consists of only two heavy chains $(\operatorname{IgH})$ and lacks the two light chains usually found in conventional $\mathrm{Abs}^{8}$. Camelids, such as camels, dromedaries and alpacas, are the only mammals that produce HCAbs. We searched the bactrian camel genome using the sequences of human, alpaca and dromedary $\operatorname{IgH}$ genes. In total, $17 \mathrm{VH}$ (heavy-chain variable region), $7 \mathrm{DH}$ (diversity region), $6 \mathrm{JH}$ (joining region) and $10 \mathrm{CH}$ (constant region) genes were identified in 16 scaffolds (Supplementary Table S14). On the basis of a unique gene cluster in scaffold 355.1, we inferred an organization of the IgH loci similar to the typical mammalian VnDn-Jn-Cn translocon ${ }^{38}$ (Fig. 3c). It has been reported that aminoacid substitutions in the sites of $37,44,45$ and 47 in the FR2 region of $\mathrm{VH}$ genes can result in conformational changes of the heavy chains, making them no longer able to bind to the light chains $^{39}$. We found that five of the $\mathrm{VH}$ genes in the bactrian camel contain such mutation sites, which may code for HCAbs (Supplementary Table S15 and Supplementary Fig. S7a). A total of six $\mathrm{C} \gamma$ genes were identified in bactrian camels, two of which have a 'GT' to 'AT' mutation on the donor splicing site in the $\mathrm{CH} 1$ region (Supplementary Table S15 and Supplementary Fig. S7b). This leads to another hallmark of the HCAbs-the lack of $\mathrm{CH} 1$ regions ${ }^{40}$.

\section{Discussion}

Our comparative analysis based on the genome sequences provides important insights into the evolution and domestication 
of bactrian camels. In addition, the identified genes and pathways may enhance our understanding of the genetic mechanisms that enable camels to survive in extreme environments. Further studies could focus on the molecular functions of the potential genes necessary for the special physiology of camels. These findings may also improve our understanding of metabolismrelated diseases, thereby benefiting human health.

\section{Methods}

Sampling, genome sequencing and assembly. An 8-year-old male wild bactrian camel named 'Naran' was selected for the wild camel sequencing on 18 February 2010. The pedigree shows that it is from a pure bred wild camel group living in the Mongolian Wild Camel Protection Area, Bayan Tooree, Altai province, Mongolia. A 6-year-old male Alashan domestic bactrian camel from Altan ovoo balgas, Alshaa aimag, Inner Mongolia, China, was selected for the domestic camel sequencing on 8 March 2010. The blood from the ear tissue was collected for both bactrian camels. The genomic DNA was extracted using Puregene Tissue Core Kit A (Qiagen).

The 500-bp paired-end and 3-kb mate-pair DNA library were sequenced on the Illumina Genome Analyzer IIx system. The 10-kb mate-pair DNA library was sequenced on the Applied Biosystems SOLiD 3 system. The $20-\mathrm{kb}$ mate-pair DNA library was sequenced using the Roche Genome Sequencer FLX system. Library preparation, sequencing and base calling were performed according to the manufacturer's recommendations.

The genome sequence of the wild camel was assembled using SOAPdenovo ${ }^{9}$ (http://soap.genomics.org.cn). We first assembled the reads with the insert size of $500 \mathrm{bp}$ into contigs using the sequence overlap information, and then used the mate-pair libraries, step by step from the shortest to the longest insert size, to join the contigs into scaffolds. To fill the intra-scaffold gaps, we retrieved read pairs that had one read well-aligned on the contigs and the other read located in the gap region.

We estimated the genome size of the wild bactrian camel based on the frequency distribution of the 17 base oligonucleotides. The reads with the insert size of $500 \mathrm{bp}$ were used in this analysis. The occurrences of 17 -mer in the reads were counted by Jellyfish $^{41}$. The peak of the k-mer frequency $(M)$ in reads is correlated with the real sequencing depth $(N)$, read length $(L)$ and k-mer length $(K)$ according to the formula $^{42} M=N \times(L-K+1) / L$. We divided the total length of all reads by the real sequencing depth, and obtained an estimated genome size of $2.38 \mathrm{~Gb}$. We also collected the $C$ values and genome sizes of other mammals to estimate the genome size of camel (http://www.genomesize.com). The camel genome has a $C$ value between 2.41 and 2.86 picograms, which is translated to a genome size between 2.02 and $2.40 \mathrm{~Gb}$.

Genome annotation. The repeat sequences were identified by RepeatMasker (version 3.2.9) (http://www.repeatmasker.org) against the Repbase4 TE library (version 2009-06-04). The transfer RNAs (tRNAs) were predicted by tRNAscanSE-1.23 (ref. 43), and short interspersed element-masked tRNAs and pseudotRNAs were eliminated. The ribosomal RNAs (rRNAs) were identified by aligning the eukaryote rRNA sequences from the SILVA ${ }^{44}$ database using Blast ${ }^{45}$ with the cutoff of $e$ value $<1 \times 10^{-5}$, identity $>85 \%$ and match length $>50 \mathrm{bp}$. The microRNAs (miRNAs) were identified by aligning the miRNA precursor sequences from miRBase ${ }^{46}$ using BlastN with the cutoff of $e$ value $<1 \times 10^{-3}$, identity $>90 \%$ and match length $>70 \mathrm{bp}$. As a result, 1701 tRNAs, 602 rRNAs and 330 miRNAs were identified in the camel genome.

For protein-coding gene annotation, $a b$ initio prediction was performed by Augustus $^{16}$ and GenScan ${ }^{17}$. Augustus predicts 26,842 genes, averaging 7.0 exons per gene. The average gene length is $21,269 \mathrm{bp}$, including coding regions $1,144 \mathrm{bp}$. GenScan predicts 42,677 genes, averaging 7.8 exons per gene. The average gene length is $31,880 \mathrm{bp}$, including coding regions $1,212 \mathrm{bp}$. Homology-based prediction was performed by searching against other mammalian gene sequences by genBlast ${ }^{47}$. A reference gene set was created by merging all of the gene sets using EVidenceModeler ${ }^{48}$. We utilized InterProScan (v 4.3) ${ }^{22}$ to collect domain information and GO terms of the camel proteins. We utilized KAAS ${ }^{49}$ (KEGG Automatic Annotation Server) for KEGG pathway annotation.

Comparative genomics and evolutionary analysis. We performed $\mathrm{Blast} \mathrm{P}^{45}$ for all the camel proteins against the NCBI HomoloGene database ${ }^{23}$ to assign them to gene families. To avoid too short local alignments, the best hit should have the $e$ value $<1 \times 10^{-5}$ and coverage length longer than $1 / 3$ on both aligned proteins. The syntenic maps between the camel and other mammalian genomes were constructed by CHSMiner ${ }^{50}$, with their orthologs as anchor points. The gap size between two neighbouring ortholog pairs was set to be $<2.0 \mathrm{Mb}$. Only the syntenic blocks with $P$-value less than $5 \times 10^{-5}$ were preserved.

We used ClustalW $W^{51}$ to perform multiple alignment for proteins in each ortholog family. The phylogeny tree was constructed based on the single-copy families in mammals, with chicken proteins as outgroups. The maximum likelihood tree for each family was built by Phylip ${ }^{52}$ under the JTT model. The consense programme in Phylip was used to integrate the individual gene trees to the final supertree.
To evaluate the supertree topology, we applied the bootstrap strategy to resample from the original tree set for 100 times $^{53}$

On the basis of reconstructed mammalian supertree, we estimated the evolutionary time scales by PAML ${ }^{29}$. We used the rooted tree with the molecular clock assumption as the null hypothesis, and the unrooted tree without the assumption as the alternative hypothesis. The LRT was used to filter genes with substitution rate significantly deviated from the molecular clock assumption $(P<0.05)$. The remaining gene trees were calibrated with the divergence time points that are consistent between fossil and molecular evidence, i.e., humanchimpanzee: $5.5 \mathrm{Mya}$, human-cattle $94 \mathrm{Mya}$ and mammal-bird: $310 \mathrm{Mya}^{54}$.

The codon-level alignment of CDS sequences from human, cattle and camel was performed by RevTrans ${ }^{55}$. Afterward, the codeml programme in PAML ${ }^{29}$ was applied to estimate the $\mathrm{dN} / \mathrm{dS}$ ratio for each gene. To select the rapidly evolving genes in camels, we used the model that the branches of camel and cattle have the same $\mathrm{dN} / \mathrm{dS}$ ratio as the null hypothesis, and the model that all branches have free $\mathrm{dN} / \mathrm{dS}$ ratios as the alternative hypothesis. The LRT was used to select rapidly evolving genes in the camel lineage (FDR $<0.05)$.

SNP and heterozygosity analysis. We utilized the BWA program ${ }^{56}$ to remap the usable reads from the wild and domestic camel to the assembled scaffolds, respectively. The parameters for mapping were chosen as the seed length of 28 and the maximum occurrences for extending a long deletion of 20 . The reads that could map to multiple positions were removed in the following analysis. The candidate SNPs and small indels $(<50 \mathrm{bp})$ were retrieved by the SAMtools pipeline ${ }^{57}$ with default settings. We flagged a candidate SNP as a likely false-positive one if it exhibits the following behaviour: (1) total depth is above 400 or below 10; (2) root mean square of mapping quality is below 20; (3) depth of alternate bases is below 4; (4) $P$-value of reference and non-reference bases being evenly distributed on both strands is below $1 \times 10^{-4}$ (Fisher exact test). These thresholds were applied to both the heterozygous SNPs within the wild and domestic camel genome, and the homozygous SNPs between them.

The heterozygosity rate was estimated as the density of heterozygous SNPs for the whole genome, gene intervals, introns and exons, respectively. For the estimation of local heterozygosity rate, sliding windows of $10 \mathrm{~kb}$ that had $90 \%$ overlap between adjacent windows were used to scan the genome. The $\chi^{2}$-test was performed for each window to identify the regions where the heterozygosity rate of the domestic camel is significantly lower than that of the wild one $(P<0.05$ after Bonferroni correction). The GO enrichment analysis for genes located in the regions was performed by GOEAST ${ }^{58}$.

\section{References}

1. Bannikov, A. Wild camels of the Gobi. Wildlife 18, 398-403 (1976).

2. Yuan, G.e.a. Ecology and Conservation of Wild Bactrian Camels (Camelus bactrianus ferus). (Mongolian Conservation Coalition \& Admon Printing, 2002).

3. Peters, J. \& Driesch, A. The two-humped camel (Camelus bactrianus): new light on its distribution, management and medical treatment in the past. J. Zool. 242, 651-679 (1997).

4. Emmanuel, B. \& Nahapetian, A. Fatty acid composition of depot fats, and rumen wall of the camel (Camelus dromedarius). Comp. Biochem. Physiol. Part B: Comp. Biochem. 67, 701-704 (1980).

5. Schmidt-Nielsen, K. Desert Animals. Physiological Problems of Heat and Water. (Oxford University Press, 1964).

6. Al-Ali, A., Husayni, H. \& Power, D. A comprehensive biochemical analysis of the blood of the camel (Camelus dromedarius). Comp. Biochem. Physiol. Part B: Comp. Biochem. 89, 35-37 (1988).

7. Ali, T. A Manual for the Primary Animal Health Care Worker. (Food and Agriculture Organization of the United Nations, 1994).

8. Hamers-Casterman, C. et al. Naturally occurring antibodies devoid of light chains. Nature 363, 446-448 (1993).

9. Li, R. et al. De novo assembly of human genomes with massively parallel short read sequencing. Genome Res. 20, 265-272 (2010).

10. Lander, E. S. et al. Initial sequencing and analysis of the human genome. Nature 409, 860-921 (2001).

11. Wade, C. et al. Genome sequence, comparative analysis, and population genetics of the domestic horse. Science 326, 865-867 (2009).

12. Elsik, C. G., Tellam, R. L. \& Worley, K. C. The genome sequence of taurine cattle: a window to ruminant biology and evolution. Science 324, 522-528 (2009).

13. Waterston, R. H. et al. Initial sequencing and comparative analysis of the mouse genome. Nature 420, 520-562 (2002).

14. Lindblad-Toh, K. et al. Genome sequence, comparative analysis and haplotype structure of the domestic dog. Nature 438, 803-819 (2005).

15. Locke, D. P. et al. Comparative and demographic analysis of orang-utan genomes. Nature 469, 529-533 (2011).

16. Stanke, M. \& Waack, S. Gene prediction with a hidden Markov model and a new intron submodel. Bioinformatics 19, ii215-ii225 (2003).

17. Burge, C. \& Karlin, S. Prediction of complete gene structures in human genomic DNA. J. Mol. Biol. 268, 78-94 (1997). 
18. Al-Swailem, A. M. et al. Sequencing, analysis, and annotation of expressed sequence tags for Camelus dromedarius. PLoS One 5, e10720 (2010).

19. Wernersson, R. et al. Pigs in sequence space: a $0.66 \mathrm{X}$ coverage pig genome survey based on shotgun sequencing. BMC Genomics 6, 70 (2005).

20. The Gene Ontology Consortium. The gene ontology: enhancements for 2011. Nucleic Acids Res. 40, D559-D564 (2012).

21. Kanehisa, M., Goto, S., Sato, Y., Furumichi, M. \& Tanabe, M. KEGG for integration and interpretation of large-scale molecular data sets. Nucleic Acids Res. 40, D109-D114 (2012).

22. Quevillon, E. et al. InterProScan: protein domains identifier. Nucleic Acids Res. 33, W116-W120 (2005).

23. Sayers, E. W. et al. Database resources of the national center for biotechnology information. Nucleic Acids Res. 39, D38-D51 (2011).

24. Delsuc, F., Brinkmann, H. \& Philippe, H. Phylogenomics and the reconstruction of the tree of life. Nat. Rev. Genet. 6, 361-375 (2005).

25. Murphy, W. J., Pevzner, P. A. \& O’Brien, S. J. Mammalian phylogenomics comes of age. Trends Genet. 20, 631-639 (2004).

26. Janis, C. M., Scott, K. M. \& Jacobs, L. L. Evolution of Tertiary Mammals of North America: Terrestrial Carnivores, Ungulates, and Ungulatelike Mammals. (Cambridge University Press, 1998).

27. Kasahara, M. et al. The medaka draft genome and insights into vertebrate genome evolution. Nature 447, 714-719 (2007).

28. Rhesus Macaque Genome Sequencing and Analysis Consortium. Evolutionary and biomedical insights from the rhesus macaque genome. Science 316, 222-234 (2007)

29. Yang, Z. PAML 4: phylogenetic analysis by maximum likelihood. Mol. Biol. Evol. 24, 1586-1591 (2007).

30. Doebley, J. F., Gaut, B. S. \& Smith, B. D. The molecular genetics of crop domestication. Cell 127, 1309-1321 (2006).

31. Wiener, P. \& Wilkinson, S. Deciphering the genetic basis of animal domestication. Proc. R. Soc. B: Biol. Sci. 278, 3161-3170 (2011).

32. Elmahdi, B., Sallmann, H. P., Fuhrmann, H., von Engelhardt, W. \& Kaske, M. Comparative aspects of glucose tolerance in camels, sheep, and ponies. Comp. Biochem. Physiol. Part A: Physiol. 118, 147-151 (1997).

33. Kaske, M., Elmahdi, B., Engelhardt, W. \& Sallmann, H. P. Insulin responsiveness of sheep, ponies, miniature pigs and camels: results of hyperinsulinemic clamps using porcine insulin. J. Comp. Physiol. B 171, 549-556 (2001).

34. Muoio, D. M. \& Newgard, C. B. Mechanisms of disease: molecular and metabolic mechanisms of insulin resistance and beta-cell failure in type 2 diabetes. Nat. Rev. Mol. Cell Biol. 9, 193-205 (2008).

35. Taniguchi, C. M., Emanuelli, B. \& Kahn, C. R. Critical nodes in signalling pathways: insights into insulin action. Nat. Rev. Mol. Cell Biol. 7, 85-96 (2006).

36. Carroll, M. A. et al. Cytochrome P-450-dependent HETEs: profile of biological activity and stimulation by vasoactive peptides. Am. J. Physiol. Regul. Integr. Comp. Physiol. 271, R863-R869 (1996).

37. Zhao, X., Pollock, D. M., Inscho, E. W., Zeldin, D. C. \& Imig, J. D. Decreased renal cytochrome $\mathrm{P} 4502 \mathrm{C}$ enzymes and impaired vasodilation are associated with angiotensin salt-sensitive hypertension. Hypertension 41, 709-714 (2003).

38. Achour, I. et al. Tetrameric and homodimeric camelid IgGs originate from the same IgH locus. J. Immunol. 181, 2001-2009 (2008).

39. Vu, K. B., Ghahroudi, M. A., Wyns, L. \& Muyldermans, S. Comparison of llama $\mathrm{VH}$ sequences from conventional and heavy chain antibodies. Mol. Immunol. 34, 1121-1131 (1997).

40. Nguyen, V. K., Hamers, R., Wyns, L. \& Muyldermans, S. Loss of splice consensus signal is responsible for the removal of the entire $\mathrm{C}(\mathrm{H}) 1$ domain of the functional camel IGG2A heavy-chain antibodies. Mol. Immunol. 36, 515-524 (1999).

41. Marcais, G. \& Kingsford, C. A fast, lock-free approach for efficient parallel counting of occurrences of k-mers. Bioinformatics 27, 764-770 (2011).

42. Li, R. et al. The sequence and de novo assembly of the giant panda genome. Nature 463, 311-317 (2009).

43. Lowe, T. M. \& Eddy, S. R. tRNAscan-SE: a program for improved detection of transfer RNA genes in genomic sequence. Nucleic Acids Res. 25, 955-964 (1997).

44. Pruesse, E. et al. SILVA: a comprehensive online resource for quality checked and aligned ribosomal RNA sequence data compatible with ARB. Nucleic Acids Res. 35, 7188-7196 (2007)

45. Altschul, S. F. et al. Gapped BLAST and PSI-BLAST: a new generation of protein database search programs. Nucleic Acids Res. 25, 3389-3402 (1997).

46. Griffiths-Jones, S., Saini, H. K., Van Dongen, S. \& Enright, A. J. miRBase: tools for microRNA genomics. Nucleic Acids Res. 36, D154-D158 (2008).

47. She, R., Chu, J. S. C., Wang, K., Pei, J. \& Chen, N. genBlastA: Enabling BLAST to identify homologous gene sequences. Genome Res. 19, 143-149 (2009).
48. Haas, B. J. et al. Automated eukaryotic gene structure annotation using EVidenceModeler and the Program to Assemble Spliced Alignments. Genome Biol. 9, R7 (2008).

49. Moriya, Y., Itoh, M., Okuda, S., Yoshizawa, A. C. \& Kanehisa, M. KAAS: an automatic genome annotation and pathway reconstruction server. Nucleic Acids Res. 35, W182-W185 (2007).

50. Wang, Z., Ding, G., Yu, Z., Liu, L. \& Li, Y. CHSMiner: a GUI tool to identify chromosomal homologous segments. Algorithms Mol. Biol. 4, 2 (2009).

51. Chenna, R. et al. Multiple sequence alignment with the Clustal series of programs. Nucleic Acids Res. 31, 3497-3500 (2003)

52. Felsenstein, J. Inferring phylogenies from protein sequences by parsimony, distance, and likelihood methods. Methods Enzymol. 266, 418-427 (1996).

53. Burleigh, J. G., Driskell, A. C. \& Sanderson, M. J. Supertree bootstrapping methods for assessing phylogenetic variation among genes in genome-scale data sets. Syst. Biol. 55, 426-440 (2006).

54. Blair Hedges, S. \& Kumar, S. Genomic clocks and evolutionary timescales. Trends Genet. 19, 200-206 (2003).

55. Wernersson, R. \& Pedersen, A. G. RevTrans: multiple alignment of coding DNA from aligned amino acid sequences. Nucleic Acids Res. 31, 3537-3539 (2003).

56. Li, H. \& Durbin, R. Fast and accurate short read alignment with BurrowsWheeler transform. Bioinformatics 25, 1754-1760 (2009).

57. Li, H. et al. The sequence alignment/map (SAM) format and SAMtools. Bioinformatics 25, 2078-2079 (2009).

58. Zheng, Q. \& Wang, X. J. GOEAST: a web-based software toolkit for gene ontology enrichment analysis. Nucleic Acids Res. 36, W358-W363 (2008).

\section{Acknowledgements}

We are indebted to many people whose names are not included in the author list, but who contributed to this project. We thank the Ministry of Environment and Sources of Mongolia for permitting sample collections from wild bactrian camels. This work was supported by State key basic research programme (973) (2011CB910204, 2010CB529206, 2011CBA00801), Research Programme of CAS (KSCX2-EW-R-04, KSCX2-YW-R-190, 2011KIP204), National Natural Science Foundation of China (30900272, 31070752), Chinese Ministry for Science and Technology Grant 2008BAI64B01, Chinese High-Tech R\&D Programme (863) (2009AA02Z304) and SA-SIBS Scholarship Programme.

\section{Author contributions}

., Z.W., G.H.D., Y.M.S., G.L.C. and Z.H.S. contributed equally to this work as first authors. J.M.L., Y.X.S., Z.X., C.H., S.H. and Y.Z. contributed equally to this work as second authors. J., H.P.Z., L.W., G.H.D., Y.X.L. and H.M. are the principal investigators and project managers in this work. J., G.L.C., M.B., W.B.Z., B.T., W., B.I.S., B.B., Z.X.W J.W., N., T., S., N., L.P., Y., G., D., E., A., T.L., M.H.C., B., H., Z.T.A. and H.P.Z conducted the sample collection and biological traits analysis; Z.H.S., Y.M.S., Z.X., J.M.L Z.L.J., Q.L., Y.C.C., F.H., G.Y.Z., F.Q., Z.K.S., L.L.Z., W.J.Z., H.M. and L.W. coordinated genome sequencing, assembly and annotation. G.H.D., Z.W., Z.X., J.M.L., C.H., B.H.L., C.L., Y.Q.C., X.Y.T., C.Y.G., W.L., L.M., T., A.Y.C., Y.L., J.H.G., J.L., H.M. and Y.X.L. did the comparative genome analysis. S.H., S.Y., J.M.L., G.H.D., Z.W., C.H., S.N., S.D.L., C.L., Y.Q.C., Z.L.J., U., Q.L., H.W.G., T.S., Z.X.Z., M.Z., C.C., T.B., T.B., J. and H.M. carried out the functional genomics analysis. Z.X. and Y.Z. submitted the genome sequence data to NCBI. Z.W., Y.X.S., Y.Z., G.H.D., Y.X.L. and H.M. wrote and edited the manuscript. Final editing of the text, Tables and Figures was done by Z.W., G.H.D., Y.X.L. and H.M.

\section{Additional information}

Accession codes: This Whole Genome Shotgun project has been deposited in DDBJ/ EMBL/GenBank as project accession PRJNA76177. The genome assembly has been deposited in DDBJ/EMBL/GenBank under the accession code AGVR00000000. The version described in this paper is the first version, AGVR01000000.

Supplementary Information accompanies this paper at http://www.nature.com/ naturecommunications

Competing financial interests: The authors declare no competing financial interests.

Reprints and permission information is available online at http://npg.nature.com/ reprintsandpermissions/

How to cite this article: Jirimutu. et al. Genome sequences of wild and domestic bactrian camels. Nat. Commun. 3:1202 doi: 10.1038/ncomms2192 (2012).

License: This work is licensed under a Creative Commons Attribution-NonCommercialShare Alike 3.0 Unported License. To view a copy of this license, visit http://creativecommons.org/licenses/by-nc-sa/3.0/ 
Jirimutu', Zhen Wang ${ }^{2,3,4}$, Guohui Ding ${ }^{2,3,4}$, Gangliang Chen ${ }^{5}$, Yamin Sun ${ }^{6}$, Zhihong Sun ${ }^{1}$, Heping Zhang ${ }^{1}$, Lei Wang ${ }^{6}$, Surong Hasi ${ }^{7}$, Yan Zhang ${ }^{8}$, Jianmei $\mathrm{Li}^{1}$, Yixiang Shi ${ }^{2,9}$, Ze Xu ${ }^{4}$, Chuan He ${ }^{10}$, Siriguleng $\mathrm{Yu}^{7}$, Shengdi Li ${ }^{3}$, Wenbin Zhang ${ }^{11}$, Mijiddorj Batmunkh ${ }^{12}$, Batsukh Ts ${ }^{13}$, Narenbatu ${ }^{14}$, Unierhu ${ }^{14}$, Shirzana Bat-Ireedui ${ }^{15}$, Hongwei Gao ${ }^{10,16}$, Banzragch Baysgalan ${ }^{17}$, Qing Li', Zhiling Jia1', Turigenbayila14, Subudenggerile7, Narenmanduhu", Zhaoxia Wang 1 , Juan Wang1, Lei Pan¹, Yongcan Chen ${ }^{10,18}$, Yaichil Ganerdene ${ }^{19}$, Dabxilt ${ }^{20}$, Erdemt $^{21}$, Altansha 22, Altansukh ${ }^{23}$, Tuya Liu ${ }^{24}$, Minhui Cao ${ }^{25}$, Aruuntsever ${ }^{26}$, Bayart ${ }^{27}$, Hosblig28, Fei He ${ }^{4}$, A Zha-ti' ${ }^{29}$, Guangyong Zheng ${ }^{3}$, Feng Qiü24, Zikui Sun ${ }^{18}$, Lele Zhao ${ }^{10}$, Wenjing Zhao ${ }^{10}$, Baohong Liu ${ }^{4}$, Chao $\mathrm{Li}^{3}$,

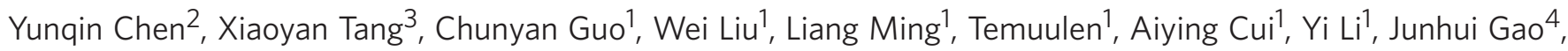
Jing $\mathrm{Li}^{2}$, Wurentaodi ${ }^{11}$, Shen $\mathrm{Niu}^{3}$, Tao Sun ${ }^{10}$, Zhengxiao Zhai ${ }^{10}$, Min Zhang ${ }^{1}$, Chen Chen ${ }^{1}$, Tunteg Baldan ${ }^{30}$, Tuman Bayaer ${ }^{31}$, Yixue Li $\mathrm{Li}^{2,3,32,33}$, He Meng ${ }^{10}$

${ }^{1}$ Key Laboratory of Dairy Biotechnology and Engineering, Ministry of Education, College of Food Science and Engineering, Inner Mongolia Agricultural University, 306 Zhaowuda Road, Huhhot 010018, China. ${ }^{2}$ Shanghai Center for Bioinformation Technology, 1278 Keyuan Road, Shanghai 201203, China. ${ }^{3}$ Key Lab of Systems Biology, Shanghai Institutes for Biological Sciences, Chinese Academy of Sciences, 320 Yueyang Road, Shanghai 200031, China.

${ }^{4}$ EG Information Technology Enterprise (EGI), Encode Genomics Biotechnology Co., Ltd., 100 Qinzhou Road, Shanghai 200235, China. ${ }^{5}$ Bactrian Camel Academe of Altai, Xingjiang, Wangyuan Camel Milk Limited Company, 99 Huanchengdong Road, Fuhai County, Xinjiang 836500, China. ${ }^{6}$ Tianjin Key Laboratory of Microbial Functional Genomics, TEDA School of Biological Sciences and Biotechnolgy, Nankai University, 23 Hongda Street, TEDA,

Tianjin 300457, China. ${ }^{7}$ Key Laboratory of Clinical Diagnosis and Treatment Technology in Animal Disease, Ministry of Agriculture, College of Veterinary Medicine, Inner Mongolia Agricultural University, 306 Zhaowuda Road, Huhhot 010018, China. ${ }^{8}$ Virginia Bioinformatics Institute, Virginia Tech, Washington Street, MC0477, Blacksburg, Virginia 24061, USA. ${ }^{9}$ School of Chemistry and Chemical Engineering, Shanghai Jiaotong University, 800 Dongchuan Road, Shanghai 200240, China. ${ }^{10}$ Shanghai Key Laboratory of Veterinary Biotechnology, School of Agriculture and Biology, Shanghai Jiaotong University, 800 Dongchuan Road, Shanghai 200240, China. ${ }^{11}$ Bactrian Camel Institute of Alsha, Inner Mongolia, 16 Tuerhute Road, Bayanhot, Inner Mongolia 750306, China. ${ }^{12}$ Mongolian Wild Camel Protection Area, Ministry of Nature and Environment, Ulaanbaatar 82050, Mongolia. ${ }^{13}$ Mongolian University of Science and Technology, Baga Toiruu 34, Sukhbatar District, Ulaanbaatar 210646, Mongolia. ${ }^{14}$ College of Animal Science, Inner Mongolia Agricultural University, 306 Zhaowuda Road, Huhhot 010018, China. ${ }^{15}$ Zelem Limited Company, 2 horoo 25-114, 2 Sukhbatar district, Ulaanbaatar 210646, Mongolia. ${ }^{16}$ Shanghai Quality Safety Center of Agricultural Products, 779 Xianxiaxi Road, Shanghai 200335, China. ${ }^{17}$ Olonlog Magazine, B-3 HD-71, Khan-Uul district, Ulaanbaatar 210431, Mongolia. ${ }^{18}$ Shanghai Personal Biotechnology Limited Company, 777 Longwu Road, Shanghai 200336, China. ${ }^{19}$ Atarchandagana Suu Limited Company, Hentii Aimag 23140, Mongolia. ${ }^{20}$ Sunid People's Government, Shiliingol Aimag, 16 Hangai Road, Achit Street, Saihantal, Inner Mongolia 011200 , China. ${ }^{21}$ Animal Husbandry Workstation of West Sunid, Shiliingol Aimag, 117 Hangai Road, Achit Street, Saihantal, Inner Mongolia 011200, China. ${ }^{22}$ Animal Husbandry Workstation of Shiliingol Aimag, 52 Eejnuur Road, Tsahar Street, Shiliinhot City, Inner Mongolia 026000, China. ${ }^{23}$ Animal Husbandry Institute of Shiliingol Aimag, 54 Shiliin Street, Shiliinhot City, Inner Mongolia 026000, China. ${ }^{24}$ Veterinary Bureau of East Alsha, 9 Ejnee Road, Bayanhot, Inner Mongolia 750300, China. ${ }^{25}$ Veterinary Bureau of East Alsha, 12 South Ring Road, Bayanhot, Inner Mongolia 750300, China. ${ }^{26}$ Animal Husbandry Workstation of East Sunid, Shiliingol Aimag, 66 Darhan Road, Mandalt, Inner Mongolia 011300, China. ${ }^{27}$ Animal Husbandry Bureau of Otog, 18 Burguud Road, Ulaan, Erdos, Inner Mongolia 016100, China. ${ }^{28}$ Animal Husbandry Bureau of North Urad, 9 Tsog Undur Road, Dunshuur, Bayannuur, Inner Mongolia 015500, China. ${ }^{29}$ Animal Science Institute, Xinjiang Academy of Animal Science, 151 Kelamayi East Street, Urumqi 830000, China. ${ }^{30}$ Research Institute of Animal Husbandry, J.Sambuu, Zaisan, Khoroo 11, Khan-Uul District, Ulaanbaatar 210153, Mongolia. ${ }^{31}$ Bureau of Agriculture and Animal Husbandry, Wula Road, West Ujumqin, Shiliingol Aimag 026200, Inner Mongolia, China. ${ }^{32}$ College of Life Science and Biotechnology, Shanghai Jiaotong University, 800 Dongchuan Road, Shanghai 200240, China. ${ }^{33}$ School of Life Sciences and Technology, Tongji University, 1239 Siping Road, Shanghai 200092, China. 


\section{Corrigendum: Genome sequences of wild and domestic bactrian camels}

The Bactrian Camels Genome Sequencing and Analysis Consortium

Nature Communications 3:1202 doi: 10.1038/ncomms2192 (2012); Published 13 Nov 2012; Updated 16 Aug 2013

Provision for access to the domestic camel genome sequence was not provided in this Article. The sequence has now been deposited in NCBI Sequence Read Archive under accession code SRX224984. 